\title{
Dynamical analysis of sea-breeze hodograph rotation in Sardinia
}

\author{
N. Moisseeva and D. G. Steyn \\ The University of British Columbia, Department of Earth, Ocean and Atmospheric Sciences, Vancouver, Canada \\ Correspondence to: N. Moisseeva (nmoisseeva@eos.ubc.ca)
}

Received: 20 June 2014 - Published in Atmos. Chem. Phys. Discuss.: 8 September 2014

Revised: 19 November 2014 - Accepted: 21 November 2014 - Published: 18 December 2014

\begin{abstract}
This study investigates the diurnal evolution of sea-breeze $(\mathrm{SB})$ rotation over an island at the middle latitudes. Earlier research on sea breezes in Sardinia shows that the onshore winds around various coasts of the island exhibit both the theoretically predicted clockwise rotation as well as seemingly anomalous anticlockwise rotation. A nonhydrostatic fully compressible numerical model (WRF) is used to simulate wind fields on and around the island on previously studied sea-breeze days, and is shown to capture the circulation on all coasts accurately. Diurnal rotation of wind is examined, and patterns of clockwise and anticlockwise rotation are identified. A dynamical analysis is performed by extracting individual forcing terms from the horizontal momentum equations. Analysis of several regions around the island shows that the direction of rotation is a result of a complex interaction between near-surface and synoptic pressure gradient, Coriolis and advection forcings. An idealized simulation is performed over an artificial island with dramatically simplified topography yet similar dimensions and latitude to Sardinia. Dynamical analysis of the idealized case reveals a rather different pattern of hodograph rotation to the real Sardinia, yet similar underlying dynamics. The research provides new insights into the dynamics underlying sea-breeze hodograph rotation, especially in coastal zones with a complex topography and/or coastline.
\end{abstract}

\section{Introduction}

Sea-breeze (SB) circulation is a mesoscale phenomenon driven by a mesoscale, horizontal pressure gradient resulting from the differential heating of land and water. Recently, with the availability of fully compressible three-dimensional non-hydrostatic numerical models, there has been a tremendous improvement in our understanding of the complex na- ture of SB and the associated nonlinear interactions on several scales, from meso- $\beta$ to micro-scale (Miller et al., 2003). Due to this complexity, numerical models present the only tool for studying the dynamics of SB circulation over real domains with complex topography. As shown by Zhang (2005), Ramis and Romero (1995), Steyn and Kallos (1992), Mahrer and Segal (1985) and Walsh (1974), the general structure and diurnal cycle of the SB circulation can be well reproduced by a numerical model, and agrees closely with the aspects developed by theoretical studies and observations.

Crosman and Horel (2010) conducted a thorough review of the existing literature on numerical studies of SBs to summarize the current state of knowledge of the dependence of SB on geophysical variables. Despite the large number of scientific studies devoted to the subject, Crosman and Horel identified several gaps in the understanding of SB circulation. Among them is the ambiguity associated with SB dependence on topography and anticlockwise hodograph rotation.

The mechanism behind the SB rotation was first explained by Haurwitz (1947) as an effect of the Coriolis force. One can therefore expect all SBs in the Northern Hemisphere and their corresponding hodographs to rotate clockwise. However, a number of cases have been identified where an apparently anomalous anticlockwise hodograph rotation (ACR) was observed. Neumann (1977), using a two-dimensional sea- and land-breeze model, showed that the rate of turning of the direction of sea and land breezes is far from uniform over the diurnal cycle. Simpson (1996) expanded on the analysis proposed by Neumann and concluded that, in general, the Coriolis force, which is the primary factor driving typical clockwise hodograph rotation (CR) in the Northern Hemisphere, is not always the most important term in the equations of motion. Kusuda and Alpert (1983) considered the issue analytically and described hodographs in terms of phase shifts. 
Using a linear model, they showed that ACR hodograph rotation can be generated by including an ACR thermal force. The switch to ACR occurs at a critical value, which is a function of friction and latitude. They also employed a simple two-dimensional model with artificial topography and found that the dominant term inducing the rotation was a combination of pressure and surface pressure gradient. In contrast to primarily analytical and idealized work, Steyn and Kallos (1992) used a three-dimensional numerical mesoscale model to study the issue in the Attic Peninsula, Greece. Their findings were in agreement with Kusuda and Alpert (1983) as well as local observations. The paper demonstrated that a balance of pressure and terrain gradient forcing is dominant, and can result in either CR or ACR. While the studies described present extremely valuable insight into the diurnal evolution of the SB, overall hodograph rotation has been largely neglected since the original study by Haurwitz (1947), according to Crosman and Horel (2010), who suggest simulating the hodograph rotation under a wide array of geophysical variables as a topic for future exploration.

Several studies have previously examined SBs in Sardinia (Dalu and Cima, 1983; Melas et al., 2000), which is located in the western Mediterranean Sea and provides an ideal platform for investigation of SB dynamics at the middle latitudes. SBs are known to be fairly frequent in the Mediterranean region, and rich meteorological data sets are readily available from fifty meteorological stations on the island, of which twelve coastal locations are shown in Fig. 1. Sardinia has a complex topography with three mountain ranges situated slightly closer to the eastern side of the island. The island is approximately elliptical in shape, with length and width $270 \mathrm{~km}$ and $140 \mathrm{~km}$, respectively.

A recent study by Furberg (2002) focused on developing a statistical climatology of SB in Sardinia based on data collected over approximately 16-26 months using the network of 12 coastal stations shown in Fig. 1. The paper examines diurnal hodograph rotation and also shows that, under appropriate conditions, SB can develop simultaneously on all coasts of Sardinia, which is in close agreement with the finding of Melas et al. (2000). More importantly, the study demonstrates using averaged wind hodographs that both CR and ACR are known to occur along the coast of the island. These findings provide an extremely useful starting point for numerical modeling of the SB circulation and hodograph rotation in Sardinia.

\section{Methods}

\subsection{Model setup}

The Weather Research and Forecast (WRF-ARW) model is used to simulate seven SB episodes during warmer months of 1997-1998 in Sardinia identified in Furberg's (2000) work. While the model offers great operational forecasting capabil- ities, effectively no options for dynamical analysis are available; this presents a serious limitation to those using WRF for research: while the model demonstrates excellent performance and accuracy, it does not allow one to investigate the dynamics driving the modeled phenomena. In order to overcome this limitation, the model code was adjusted to allow for the extraction of the individual tendency terms of the horizontal momentum equations. The details of the introduced changes, as well as modified code, are available as the Supplement.

The knowledge that the episode days identified by Furberg (2000) had favorable atmospheric conditions for the formation of SB on the island provides an extremely useful starting point for the numerical simulation. Identified primarily on the basis of diurnal reversal of surface wind direction first suggested by Steyn and Faulkner (1986), the following days were considered SB days on Sardinia:

- 17 May 1997

- 15 August 1997

- 20 May 1998

- 30 May 1998

- 20 June 1998

- 21 June 1998

- 29 June 1998

Identical domain configuration was used for all of the SB episode days. The simulation was set up on a two-way nested domain centered on the island of Sardinia with 50 vertical eta levels. The parent and nest domain grid spacings were set to 9 and $3 \mathrm{~km}$, respectively. Selection of such horizontal spacing was largely based on earlier studies by Steyn et al. (2013), which concluded that, at this resolution, the subgrid-scale effects in WRF have no significant influence on the overall dynamics of the SB.

The model was initialized using Climate Forecast System Reanalysis (CFSR) high-resolution pressure-level $\left(0.5^{\circ}\right.$ latitude/longitude $)$ and surface and radiative flux $\left(0.3^{\circ}\right.$ Gaussian grid) $6 \mathrm{~h}$ forecast data. Each of the SB days identified by Furberg (2000) was simulated over $30 \mathrm{~h}$ to account for a $6 \mathrm{~h}$ spin-up, beginning at 18:00 UTC of the previous day. As we are primarily interested in daytime dynamics, the analysis was performed starting at 09:00 UTC, i.e., $15 \mathrm{~h}$ after the beginning of each simulation. Wind and dynamical tendency fields were output six times each hour and subsequently averaged to produce an estimate of hourly values.

\subsection{Model evaluation}

The model was evaluated using the observational data available for the 12 meteorological stations operated by the Servizio Agrometeorologico Regionale per la Sardengna 


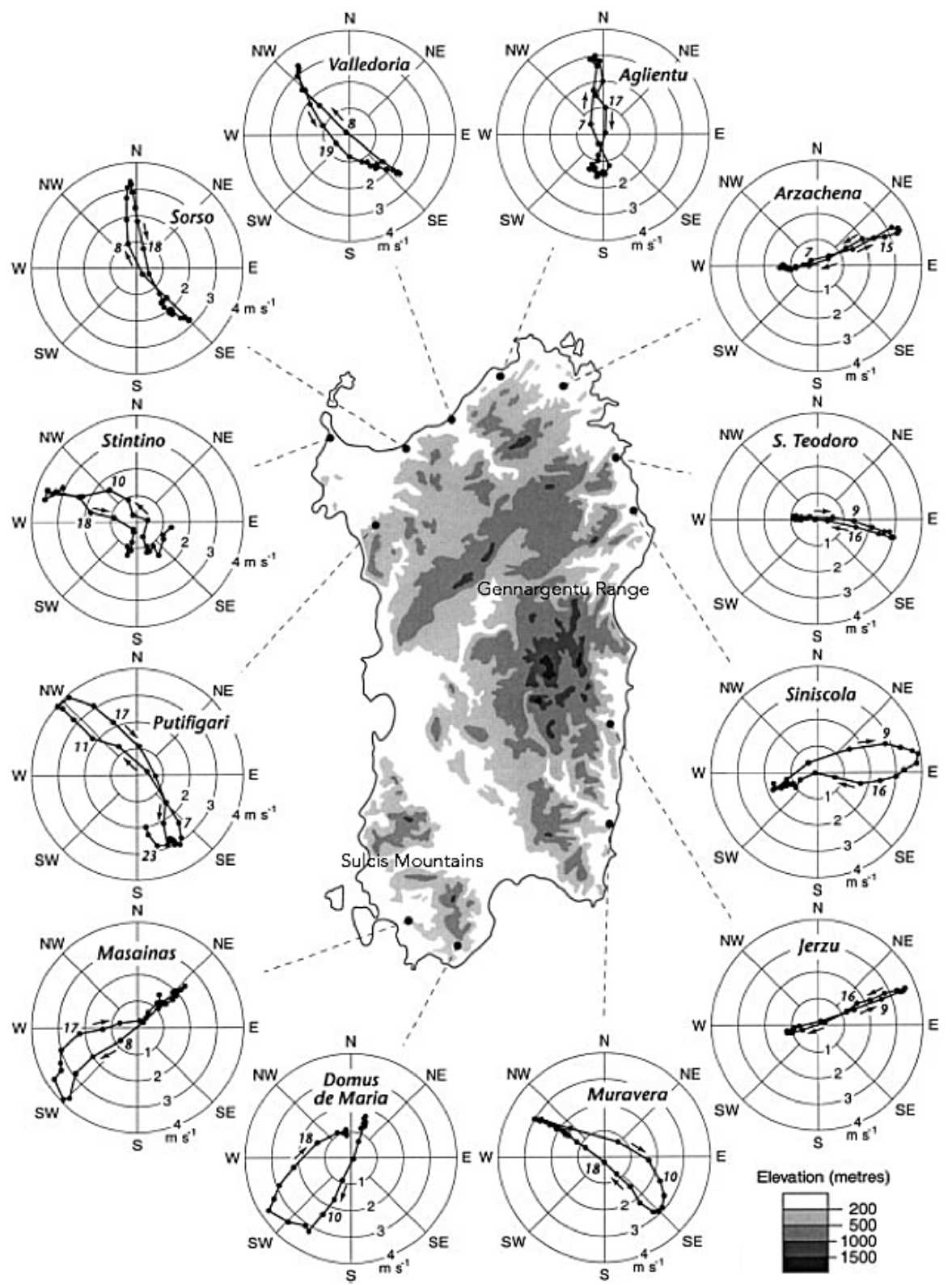

Figure 1. Topographic map of Sardinia with 12 coastal stations and SB hodographs. The island is approximately $270 \mathrm{~km}$ long and $140 \mathrm{~km}$ wide; from Furberg et al. (2002).

(SAR), who manage a network of 50 stations over the island. The primary goal was to demonstrate that the model captures the SB circulation and hodograph rotation on all coasts, and hence we focused on comparing the winds and associated diurnal evolution of wind hodographs at each of the station locations. For the purpose of the analysis, the twelve individual modeled grid points were selected on the island, which are closest to the location of the meteorological stations based on latitude and longitude.

The first step was to determine the direction of the onshore wind vector for both modeled and observational data. The $u$ and $v$ wind components at $10 \mathrm{~m}$ were converted into polar coordinates and plotted as a hodograph for both observed and modeled winds. Figure 2 shows the results from a single simulated SB episode on 21 June 1998. A range of onshore/offshore directions was tested for each data set, and the onshore wind vector was defined as the one that produced the smallest root mean square error (RMSE) between model and observation, and hence corresponding to the primary direction of air flow.

Note that the modeled and observed onshore directions were defined separately for the observed and modeled winds and allowed to differ for each individual day. While these directions were generally found to be in close agreement, 


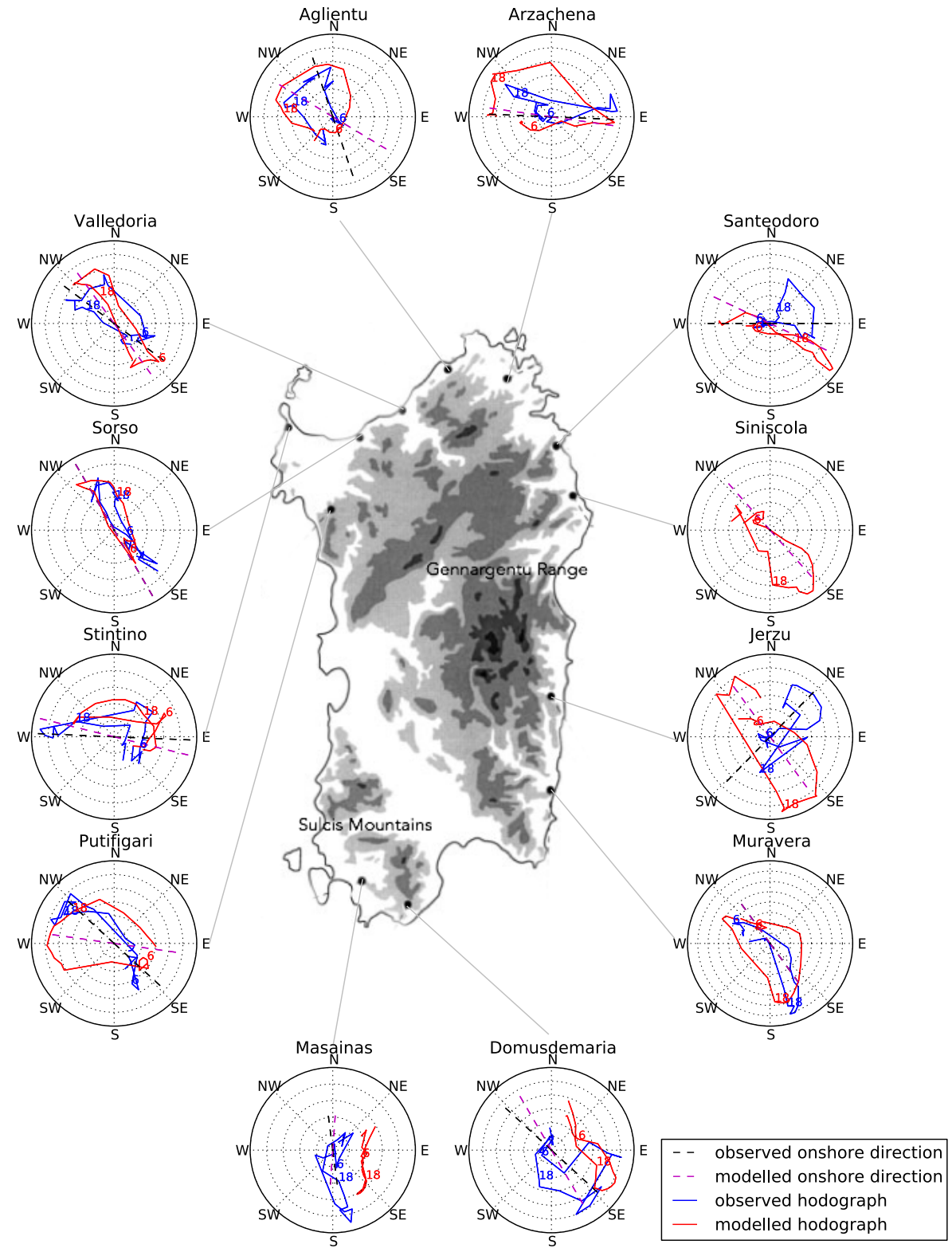

Figure 2. Modeled and observed wind hodographs at $10 \mathrm{~m}$ over Sardinia on 21 June 1998.

several stations showed a considerable discrepancy between model and observations (e.g., Valledoria, Jerzu). Introduction of this flexibility is a particularly important aspect of the proposed evaluation methodology. While it may seem that this assumption makes the evaluation criteria less stringent, it in fact acts as a filter to isolate the subgrid-scale effects that are inevitably present in observational data, and entirely absent from the model output. As the hourly winds are subsequently projected onto the identified onshore direction, a slight difference in the definition of the onshore axis due to inclusion/exclusion of the subgrid-scale effects can easily mask the model's overall good performance. The advantage of this approach is that it allows us to compare and evaluate the larger-scale kinematics and their diurnal evolution, rather than the local effects due to subgrid-scale phenomena.

Overall, individual (non-averaged) daily hodographs present one of the most stringent criteria for model evaluation, as instantaneous wind data are inevitably highly variable. However, as seen in Fig. 2, there is good agreement between modeled and observed winds. While no observational record is present for Siniscola station for the modeled day, there appears to be a greater discrepancy between simulated and observed data on the eastern coast. Modeled winds at Jerzu station, located beneath the highest peak in the Gennargentu Range (1834 m) (Fig. 1), are consistently inferior to simulations at other stations on all simulation days. This can 

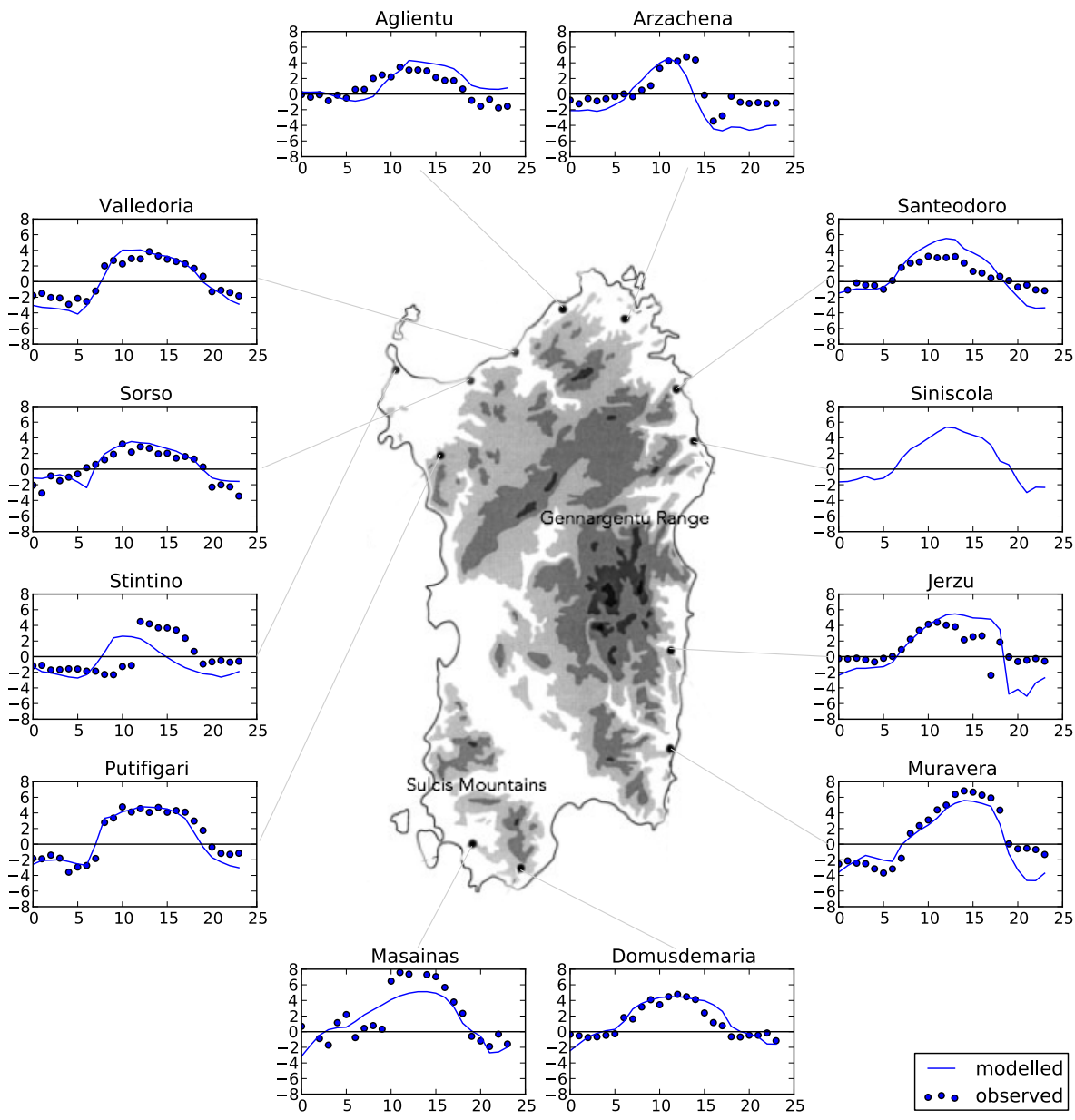

Figure 3. Diurnal evolution of onshore wind at $10 \mathrm{~m}$ over Sardinia on 21 June 1998.

likely be explained by steep topography in close proximity to the eastern coast, which presents a well-known challenge for numerical models. The nonorthogonal grid lines introduced due to the use of terrain-following vertical coordinates in WRF are known to produce significant truncation errors when attempting to calculate horizontal gradients, leading to computational inaccuracy of advection, diffusion and pressure gradient terms (Yamazaki and Satomura, 2010).

The simulated and observed wind fields were then projected onto the corresponding identified onshore direction for each station and compared. As seen in Fig. 3, the model evaluation demonstrates excellent agreement between model and observations, both due to exceptional model performance and successful evaluation strategy. Note that the stations where the onshore directions determined by RMSE differed considerably between model and observations still show strong agreement in the development and shape of the onshore wind component, suggesting that the overall structure of the SB at that location is well represented. WRF clearly captures the kinematics of the SB circulation, and hence can be used for further dynamical analysis of the SB structure.

\section{Dynamical analysis of a real-case simulation}

\subsection{Rotation of the horizontal wind}

The horizontal momentum equations solved in WRF can be represented in simplified vector form as

$$
\frac{\partial \boldsymbol{V}_{\mathrm{h}}}{\partial t}=\frac{\partial \boldsymbol{V}_{\mathrm{pg}}}{\partial t}+\frac{\partial \boldsymbol{V}_{\mathrm{adv}}}{\partial t}+\frac{\partial \boldsymbol{V}_{\mathrm{cor}}}{\partial t}+\frac{\partial \boldsymbol{V}_{\mathrm{hdif}}}{\partial t}+\frac{\partial \boldsymbol{V}_{\mathrm{vdif}}}{\partial t},
$$

where $\boldsymbol{V}_{\mathrm{h}}$ is the total horizontal velocity vector, $\boldsymbol{V}=(U, V)$ and subscripts pg, adv, cor, hdif, and vdif correspond to forcing due to pressure gradient, advection, Coriolis, and horizontal and vertical diffusion. Taking the $850 \mathrm{mb}$ pressure level to be representative of overlying synoptic weather conditions, the pressure gradient term $\boldsymbol{V}_{\text {pg }}$ can be further separated into synoptic (syn) and surface (surf) forcing by assuming that

$$
\boldsymbol{V}_{\text {syn }}=\boldsymbol{V}_{\mathrm{pg}}(850 \mathrm{mb}) \text {. }
$$


The remaining pressure gradient forcing is then assumed to be due to surface effects, i.e.,

$$
\boldsymbol{V}_{\text {surf }}=\boldsymbol{V}_{\text {pg }}-\boldsymbol{V}_{\text {syn }} \text {. }
$$

It is important to note that the formulation of WRF-ARW does not allow us to separate the pressure gradient forcing further into components to isolate the effects of topography, coastline curvature and aspect, land/sea temperature contrast, roughness and other local features.

Full horizontal momentum equations considered for dynamical analysis then become

$$
\begin{aligned}
\frac{\partial \boldsymbol{V}_{\mathrm{h}}}{\partial t} & =\frac{\partial \boldsymbol{V}_{\text {surf }}}{\partial t}+\frac{\partial \boldsymbol{V}_{\text {syn }}}{\partial t}+\frac{\partial \boldsymbol{V}_{\text {adv }}}{\partial t}+\frac{\partial \boldsymbol{V}_{\text {cor }}}{\partial t}+\frac{\partial \boldsymbol{V}_{\text {hdif }}}{\partial t} \\
& +\frac{\partial \boldsymbol{V}_{\text {vdif }}}{\partial t} .
\end{aligned}
$$

Following Neumann (1977), the tendency of horizontal wind direction can be expressed as

$$
\frac{\partial \alpha}{\partial t}=\frac{1}{\boldsymbol{V}_{\mathrm{h}}^{2}} \boldsymbol{k} \cdot\left(\boldsymbol{V}_{\mathrm{h}} \times \frac{\partial \boldsymbol{V}_{\mathrm{h}}}{\partial t}\right),
$$

where $\alpha$ is the angle of local wind relative to the positive $x$ axis, $\boldsymbol{V}_{\mathrm{h}}$ is the horizontal wind vector, and $\boldsymbol{k}$ is a vertical unit vector. Positive and negative values of $\frac{\partial \alpha}{\partial t}$ correspond to ACR and CR, respectively. Expanding the cross product in Eq. (5) using the components of the total wind vector in Eq. (4), it is possible to compare the magnitudes of the terms contributing most strongly to the rotation.

\subsection{Regional patterns of hodograph rotation}

Using the definition of $\frac{\partial \alpha}{\partial t}$ in Eq. (5), it is possible to create contour maps of regions of CR and ACR for the simulated real domain. 20 June 1998 was selected as a test case, as it has shown strong agreement between model and observed hodographs based on the results of model evaluation. Hourly contours produce extremely complex patterns. As we are primarily interested in identifying possible invariant features of the circulation, daytime hourly $\frac{\partial \alpha}{\partial t}$ values were averaged between 09:00 and 17:00 UTC to produce $\frac{\partial \alpha_{\text {day }}}{\partial t}$. Figure 4 shows daytime regional patterns of $\mathrm{CR}\left(\frac{\partial \alpha_{\text {day }}}{\partial t}<0\right)$ and $\operatorname{ACR}\left(\frac{\partial \alpha_{\text {day }}}{\partial t}>0\right)$ for the real-case simulation.

The southwestern region of the nested domain is dominated by strong $\mathrm{CR}$. The $\mathrm{CR}$ pattern continues around the northern part of Sardinia. The SB circulation on the eastern coast of the island is largely ACR. The southern tip of Sardinia shows a switch between CR and ACR likely due to the presence of the southern mountain range - the Sulcis Mountains (Fig. 1). Overall, the rotation pattern appears to respond to the local features of the terrain. The island of Corsica to the north of Sardinia exhibits a very complex pattern with several extremely sharp gradients in rate of change of direction. This can likely be explained by Corsica's much steeper

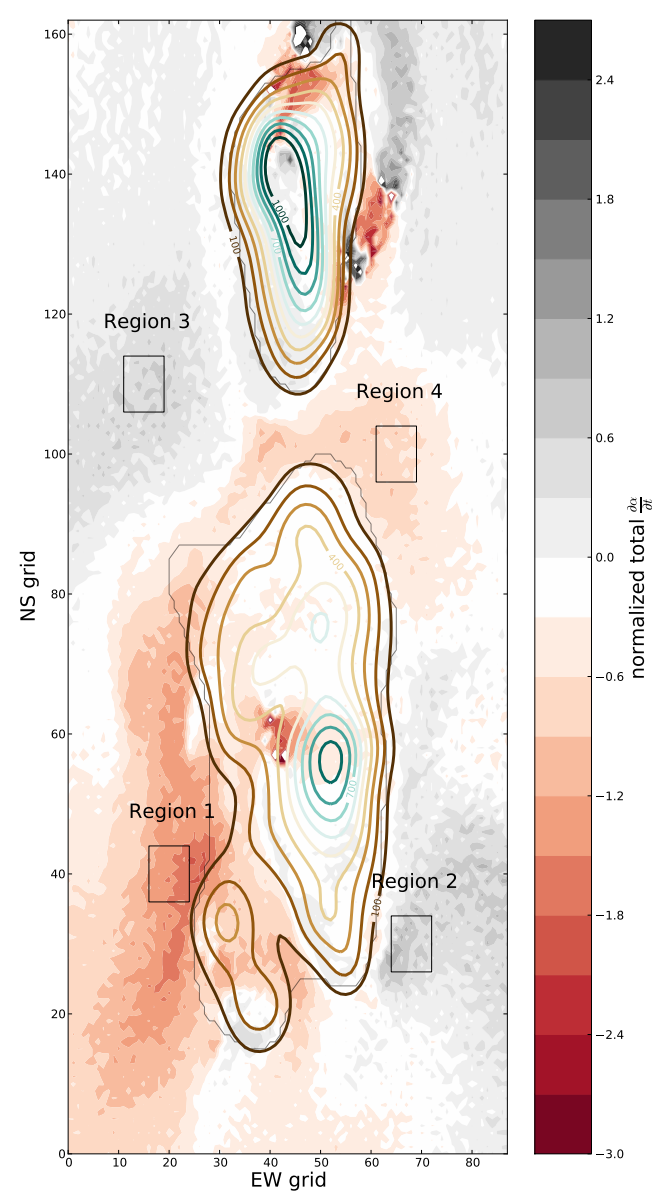

Figure 4. Total $\frac{\partial \alpha}{\partial t}$, averaged over daytime hours. Also shown are smoothed elevation contours and smoothed coastline. Regions 1, 2, 3 and 4 are identified for further dynamical analysis.

topography and slopes and proximity to the boundary of the nested domain.

Also identified in Fig. 4 are four subregions off the southwestern, northwestern, southeastern and southwestern coasts of the island exhibiting either CR or ACR. Each subregion is a square of $8 \times 8$ grid points covering an area of $576 \mathrm{~km}^{2}$. The chosen locations correspond to the largest positive and negative values of average diurnal rotational forcing, as they maintain their sense of rotation throughout the length of the day. These regions were selected for further dynamical analysis in an attempt to understand the underlying processes most strongly influencing the sense of wind rotation.

\subsection{Components of horizontal wind rotation}

Expanding the cross product in Eq. (5) using the components of the total wind vector in Eq. (4), the total rate of rotation can be broken up into individual forcing terms as follows:

$\frac{\partial \alpha_{\mathrm{tot}}}{\partial t}=\frac{\partial \alpha_{\mathrm{surf}}}{\partial t}+\frac{\partial \alpha_{\mathrm{syn}}}{\partial t}+\frac{\partial \alpha_{\mathrm{cor}}}{\partial t}+\frac{\partial \alpha_{\mathrm{adv}}}{\partial t}+\frac{\partial \alpha_{\mathrm{hdif}}}{\partial t}+\frac{\partial \alpha_{\mathrm{vdif}}}{\partial t}$, 
where the subscripts correspond to the various tendency components, consistent with terms in Eq. (4). As described earlier, the Coriolis term always induces CR in the Northern Hemisphere. However, the remaining rotation tendency components have much more complex underlying physics, and are discussed individually below.

Surface pressure gradient: the surface pressure gradient is predominantly driven by the temperature contrast between land and water, and its influence on the sense of rotation depends largely on the location and shape of the landmass. More subtle, local effects of the sea surface temperature inhomogeneity may further alter the turning direction of the surface wind. Moreover, non-uniform surface heating due to the topography and irregular coastline of Sardinia are likely to introduce further complexity into surface pressure distribution, as previously suggested by Kusuda and Alpert (1983).

Synoptic pressure gradient: as the synoptic pressure gradient term is derived from the total pressure gradient, it inevitably represents a total of upper level forcings and true synoptic flow. While the synoptic component of the pressure gradient force generally acts in opposition to the surface pressure gradient under SB conditions due to the formation of the SB return flow (Miller et al., 2003), it also responds to the local topography. Cyclonic and anti-cyclonic rotation may be induced, depending on the direction of the local wind and shape of the topography. As SBs are known to develop on all coasts of Sardinia and have variable inland penetration, the direction of dominant wind flowing over the primary mountain range on the island is likely to vary throughout the day.

Advection: advection of the horizontal momentum may similarly result in the formation of both CR and ACR. The importance of the term depends largely on the presence of velocity gradients at a given location. Since the regions selected for our analysis are located away from the coast, we can expect the rotational effects of the advection to be of secondary importance.

Horizontal and vertical diffusion: the horizontal and vertical diffusion are friction-driven effects, and hence always act to oppose the local wind. Varying surface roughness due to the spatial distribution of land use, cover and topography may introduce shear and rotation into wind flow. As our analysis is performed for locations away from the coast, these remain largely negligible.

\subsection{Relative importance of tendency terms}

To apply the term-by-term analysis (Eq. 6) to each region identified in Fig. 4, the hourly tendency values for the selected grid points were extracted. They were subsequently normalized by the Coriolis parameter $f$ to produce nondimensional values, and also spatially averaged amongst the 64 grid points for each hour. Figure 5 shows the diurnal evo- lution of the individual terms as well as their spatial standard deviation.

For Region 1, shown in Fig. 5, the total clockwise rotation rate increases throughout the day, reaching a peak around 15:00 UTC, as may be explained by the growth of the landsea temperature contrast. As expected, the Coriolis component is one of the leading terms inducing CR, and remains constant. The surface pressure gradient becomes increasingly important through the day, initially contributing slightly to ACR, but subsequently turning strongly CR. Note that the surface pressure gradient term includes the combined effects of surface slope, roughness, temperature and pressure gradient, and hence is non-zero even though the sub-domain is located over water.

The synoptic gradient acts largely in opposition to the surface pressure gradients, likely due to the formation of SB return flow near the $850 \mathrm{mb}$ level. As expected, this forcing is strongest around 1400. Since the SB return flow often merges with the prevailing synoptic flow and is, hence, difficult to distinguish (Melas et al., 2000), it is important to emphasize that true synoptic winds do not appear to be a significant factor in determining the rotation of the SB. The analysis is based on seven SB episodes that were pre-selected by Furberg (1992) using a fairly conservative filter, ensuring the exclusion of cases with strong overriding synoptic-scale winds. The model, similarly, produces very weak synoptic flow and, as evident from Fig. 5, weak synoptic rotational forcing.

The advection effect is always clockwise, but is of secondary importance in this dynamical balance. Horizontal and vertical diffusion terms are largely insignificant in this and the remaining regions, as they were located off the coast where friction is of secondary importance. As SBs are mesoscale phenomena, their scale is not restricted to the immediate coastal region. Hence, the analysis can be performed away from the regions of sharp gradients in topography, roughness, and temperature and still capture the dynamics of the phenomenon. Overall, the balance appears to be dominated by surface and synoptic pressure gradients and Coriolis.

While the Coriolis term remains one of the largest in magnitude, acting clockwise for Region 2, the combined effects of surface and synoptic pressure gradients outweigh its influence and induce ACR. Similarly to Region 1, a relatively small clockwise advection term is present. While clockwise Coriolis forcing remains the largest term throughout the course of the day, the combined anticlockwise effects of all the remaining terms induce ACR in Region 3. A particular feature of the dynamics of this region is that the advection term is relatively important, and without it the ACR would not be possible. Region 4 exhibits largely CR, dominated by the combined effect of Coriolis and pressure gradient terms. Advection and synoptic pressure gradient terms act to induce ACR, but remain secondary. 

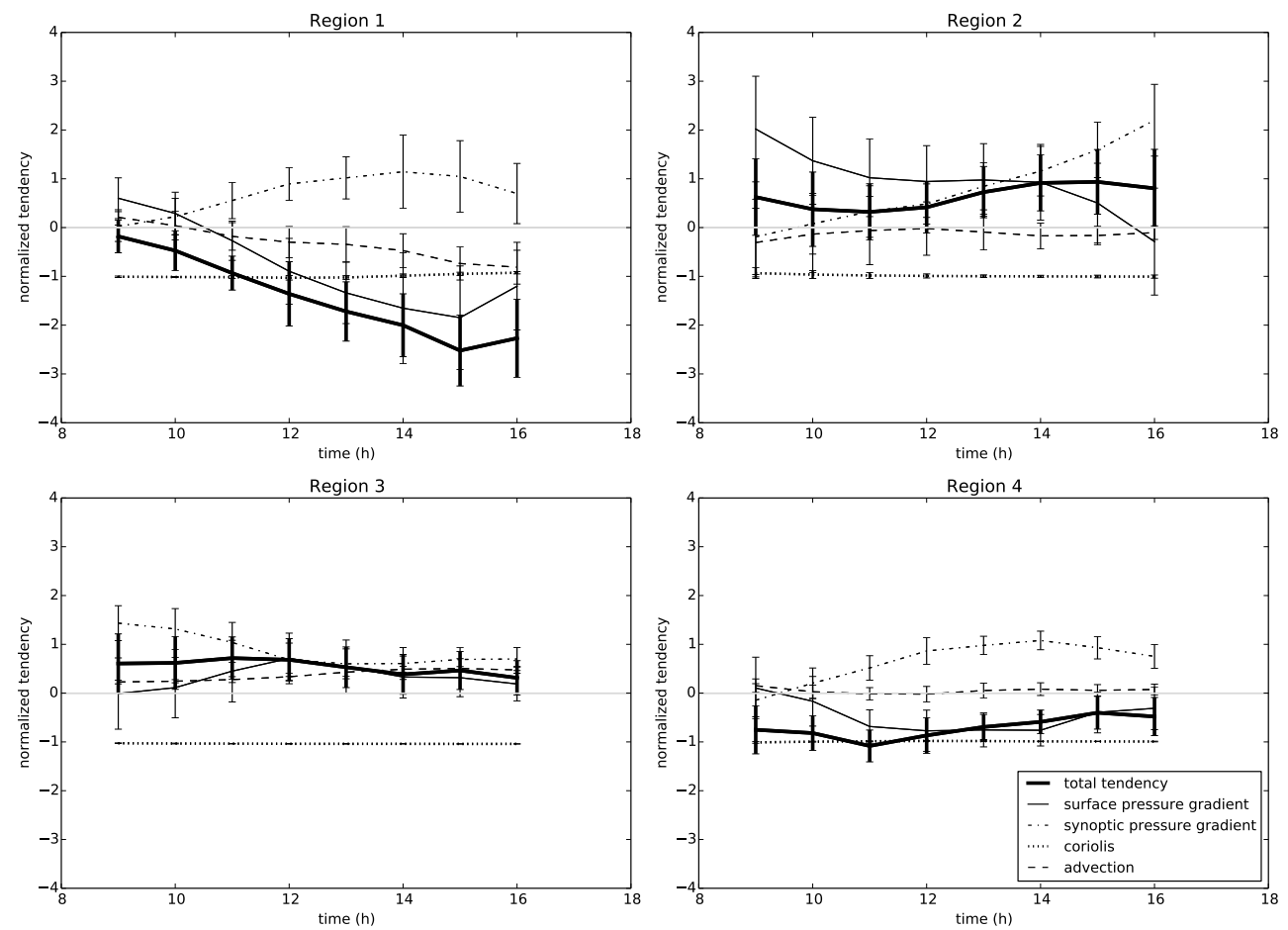

Figure 5. Evolution of dominant dynamic forcings for regions 1, 2, 3 and 4, on 20 June 1998. Positive and negative values correspond to ACR and CR, respectively.

It can hence be concluded that the sense of rotation is predominantly a result of local balance of Coriolis, surface and synoptic pressure gradients and advection. While Coriolis forcing remains constant throughout the day, the remaining tendencies each have a unique diurnal pattern. Surface pressure gradient rotation tendency appears to respond faster to the increase in the land-sea temperature contrast, generally peaking in magnitude around noon. The forcing due to the synoptic pressure gradient appears to peak later in the day, which may be explained by the formation of the SB return flow, generally forming with a slight delay in response to the surface SB gravity wave (Miller et al., 2003). The advection term is largest around 15:00 UTC, and can act to induce both clockwise and anticlockwise rotation.

Hence, the dynamical balance of terms contributing to SB rotation in Sardinia appears to be much more complicated than that proposed by Steyn and Kallos (1992) for the Attic Peninsula. The rotation tendencies due to pressure gradients were not typically found to be the largest magnitude terms. Moreover, depending on the specific region of the domain, these terms could be acting in the same or opposite directions. This work has shown that, rather than being the result of a simple balance of the synoptic and surface pressure gradients, the sense of direction is determined by whether the combined effects of surface, synoptic and advection tendencies outweigh the Coriolis effect.
The spatial pattern has also proved to be much more complex than that found in Steyn and Kallos (1992). One can speculate that the rotation patterns found around Corsica, which, while having a significantly steeper topography, closely resembles the single-hill structure of the Attic Peninsula, show a similar "CR tongue" formation near the eastern coast as found by Steyn and Kallos. However, the accuracy of the simulation was not evaluated in that region, and the proximity to the boundaries of the nest domain does not allow us to draw such conclusions with certainty. The complexity of the spatial patterns of SB rotation in Sardinia may also be explained by the increased ability of newer numerical models to capture detail. An idealized simulation was hence set up to determine whether the complexity of hodograph rotation patterns in Sardinia is associated with the specific topographic features of the island or improved numerical modeling abilities.

\section{Dynamics of an idealized case}

It is important to emphasize that the idealized case referred to herein is not to be confused with ideal-case WRF simulations, which introduce severe simplification into the model's operation. For this study, the full features of a real-case WRF simulation were retained (including all surface, boundary layer and physics routines, as well as real earth features such as curvature and Coriolis). The term idealized in this case is 
meant to indicate that the shape, coastline and topography of the island were generalized to such an extent that they no longer represent the particular characteristics of Sardinia, but rather any elliptical island with a bell-shaped topography at the middle latitudes.

Using the standard WRF Preprocessing System (WPS) software to create a domain, the idealized case study was set up with the same parameters and nesting options as the real simulation. The topography was then manually edited to create a perfectly elliptical island in the center of the domain with a 40 grid-point semi-major axis and an 18 grid-point semi-minor axis, which approximately matches the size of Sardinia. The remaining grid points of the nest and parent domains were masked as water. All other standard fields such as land use, soil moisture and type, temperature and pressure were also adjusted. The topography height was edited to create a bell-shaped mountain with an $800 \mathrm{~m}$ peak in the center of the island using a two-dimensional Hanning window.

Meteorological data from 20 June 1998 were used to create initial fields with WPS. These were again manually altered to fit the modified domain. Combined domain (including both static and meteorological fields) and boundary files generated by WRF had to be further adjusted to remove any inconsistencies arising due to interpolation. The rest of the simulation was performed using settings identical to the real cases. Since WRF was not designed to introduce idealized topography into a real-case simulation, the results presented in this section cannot be considered conclusive. The complexity of the WRF model makes tracking potential inconsistencies of these modifications extremely difficult. While the hourly patterns of hodograph rotation of the idealized simulation showed distributions nearly as complex as the realcase simulation, the diurnal average shown in Fig. 6 does exhibit a much simpler pattern than that of a real case. It is worth noting that regions of CR and ACR are arranged on opposite coasts to those of the real Sardinia, and similarly to Corsica from the real simulation and the Attic Peninsula from Steyn and Kallos (1992). This coarse pattern of CR and ACR rotation can likely be attributed to similarities between the shape and elevation of the gross topography of the Attic Peninsula and the gross topography of the idealized simulation. A consequence of this is that the more complex CR and ACR patterns in the real simulations are products of quite local topographic influences.

Overall, the structure appears to be vortex-like, centering around the peak of the mountain. One can speculate that the region of $\mathrm{CR}$ on the eastern coast of the island is the equivalent of the "CR tongue" feature of the Attic Peninsula. Again, further model analysis would be necessary to confirm this finding.

The term-by-term dynamical analysis for the idealized simulation is also much less conclusive than that of the real simulation. The western coast ACR region shows consistent but relatively weak total rotation forcing, as seen in Fig. 7 . Early morning values appear to be unrealistically large. This

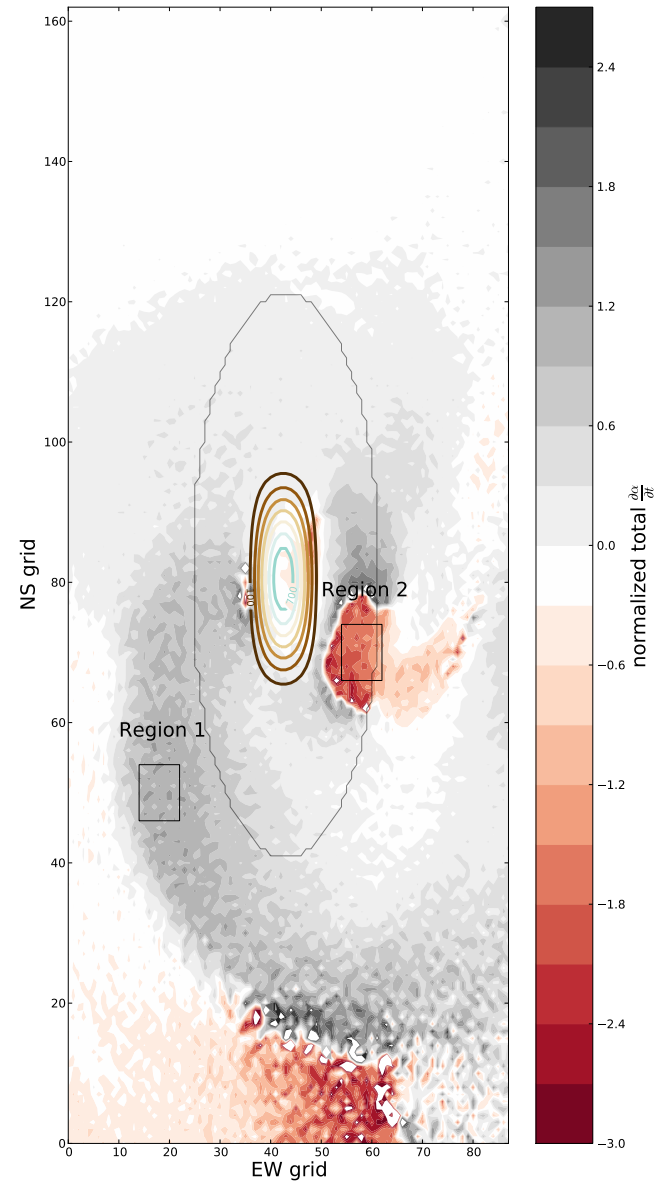

Figure 6. Total $\frac{\partial \alpha}{\partial t}$ values are averaged over daytime hours. Also shown are elevation contours and the coastline. Regions 1 and 2 are identified for further dynamical analysis.

may be an indication of a model response to the morning switch in the direction of surface heat flux, which in some cases produces a spike in model fields. As likely inconsistencies in the domain setup due to modification of the standard preprocessing routine may reduce the numerical stability of the model, the increased values caused by a spike may persist for long periods of time. Overall, the dynamics of the ACR region mimic those of Region 3 in the real-case simulation, with all significant terms acting to counterbalance Coriolis forcing. Since the idealized Region 1 remains away from the coast, the diffusion terms are again insignificant. Unlike the real-case simulation, the afternoon surface, synoptic pressure gradients and advection are all of approximately the same magnitude.

The CR region on the eastern coast (Region 2) of the idealized island shows a much stronger rotational tendency than that on the western coast. The signs of surface and synoptic pressure gradient vary throughout the day. Similarly to the idealized Region 1, pressure gradient and advection terms have alike magnitudes. As this region partially covers land 

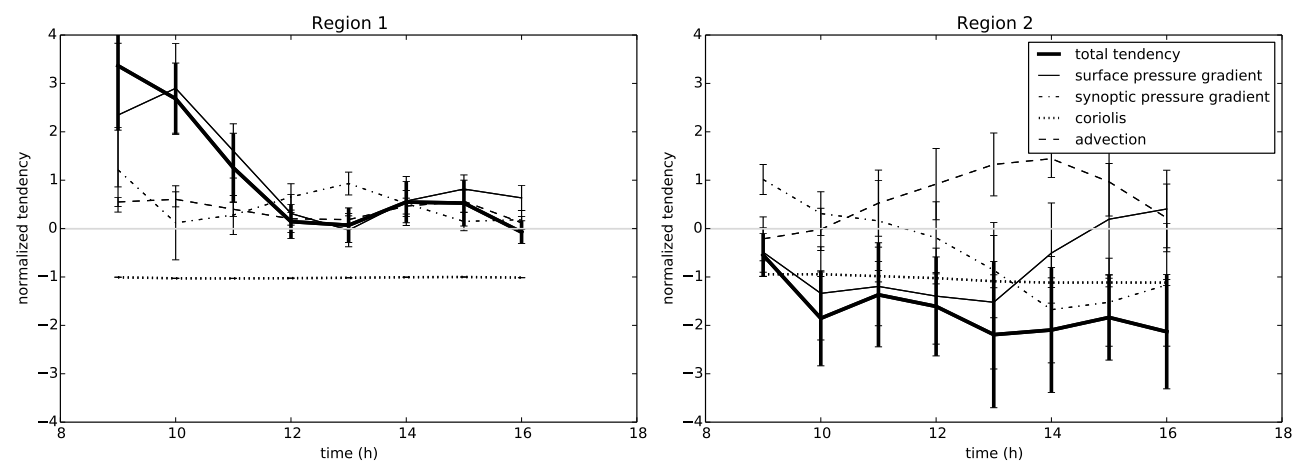

Figure 7. Evolution of dominant dynamic forcings for regions 1 and 2 (idealized case). Positive and negative values correspond to ACR and CR, respectively.

grid points, the vertical diffusion term can no longer be ignored. Friction effects contribute to the total balance, inducing both clockwise and anticlockwise rotation, depending on the time of the day. Similarly to the real case, the overall shape of the total diurnal rotation curve appears to be strongly influenced by that of the surface pressure gradient. Though Region 1 of the idealized simulation has a similar spatial variability to that of the real-case simulation, larger standard deviation values were observed for the second region.

While these results generally appear to be in agreement with the previous studies, further evaluation is necessary to deem the findings conclusive. The patterns of clockwise and anticlockwise hodograph rotation are notably simpler than those of a real-case simulation, as anticipated. While the distribution of CR and ACR regions bears little resemblance to that of the real Sardinia, the underlying dynamical behavior appears to be similar.

\section{Conclusions}

This study examined the dynamics of SB rotation in a region of complex topography, with known occurrence of both theoretically expected CR as well as "anomalous" counterCoriolis ACR. A numerical simulation was performed for Sardinia, using the WRF model and subsequently evaluated for accuracy using local observations. The original WRFARW code was adjusted to allow for the extraction of the individual components of the horizontal momentum equations from the solver. Observed and simulated daily hodographs were compared, and an evaluation methodology was devised to asses WRF's ability to capture SB kinematics. The diurnal evolution of modeled and observed onshore winds was shown to be in strong agreement.

Terms found to have a significant contribution to the total momentum balance over the domain included pressure gradient (subsequently separated into surface and synoptic components), Coriolis, advection, and horizontal and vertical diffusion. The rate of rotation of the total horizontal mo- mentum tendency was plotted for the entire domain. Following Kusuda and Alpert (1983), the strength of rotation due to each component of the horizontal momentum equations was determined for the selected regions around the island. The direction of rotation was found to be a result of a complex interaction between surface and synoptic pressure gradients, Coriolis and advection.

Lastly, an idealized simulation was attempted using a similar domain configuration as that of a real case, but introducing a completely artificial simplified topography. Contour maps of regions of CR and ACR showed the formation of a vortex-like region of ACR around most of the island, with a single protruding "tongue" of the CR region in the middle of the eastern coast. The dynamical analysis of regions of CR and ACR showed that the balance of forces resembled those of the real simulation; however, higher variability, as well as unlikely individual term magnitudes, suggests that the simulation requires further improvements to be considered conclusive.

\section{The Supplement related to this article is available online at doi:10.5194/acp-14-13471-2014-supplement.}

Acknowledgements. The authors would like to thank Alessandro Delitala and Maria Furberg for their help with SAR data. Reanalysis fields were provided by National Centers for Environmental Prediction (NCEP) and the Computational and Information Systems Laboratory (CISL). Special thanks to Peter Jackson, Jim Dudhia and Anthony Toigo for their remote assistance with WRF. This work was funded by the Natural Sciences and Engineering Research Council of Canada (NSERC) and the IACPES CREATE program.

Edited by: S. Galmarini 


\section{References}

Crosman, E. and Horel, J.: Sea and Lake Breezes: A Review of Numerical Studies, Bound.-Lay. Meteorol., 137, 1-29, 2010.

Dalu, G. and Cima, A.: Three-dimensional airflow over Sardinia, Il Nuovo Cimento, 6, 453-472, 1983.

Furberg, M.: Sea breezes on Sardinia, Master's thesis, Uppsala University, Sweden, 2000

Furberg, M., Steyn, D., and Baldi, M.: The climatology of sea breezes on Sardinia, Int. J. Climatol., 22, 917-932, 2002.

Haurwitz, B.: Comments on the sea-breeze circulation, J. Meteorol., 4, 1-8, 1947.

Kusuda, M. and Alpert, P.: Anti-Clockwise Rotation of the Wind Hodograph. Part I: Theoretical Study, J. Atmos. Sci., 40, 487499, 1983

Mahrer, Y. and Segal, M.: On the Effects of Islands' Geometry and Size on Inducing Sea Breeze Circulation, Mon. Weather Rev., 113, 170-174, 1985.

Melas, D., Lavagnini, A., and Sempreviva, A.: An Investigation of the Boundary Layer Dynamics of Sardinia Island under SeaBreeze Conditions, J. Appl. Meteorol., 39, 516-524, 2000.

Miller, S., Keim, B., Talbot, R., and Mao, H.: Sea breeze: Structure, forecasting, and impacts, Rev. Geophys., 41, 1011, doi:10.1029/2003RG000124, 2003.
Neumann, J.: On the Rotation Rate of the Direction of Sea and Land Breezes, J. Atmos. Sci., 34, 1913-1917, 1977.

Ramis, C. and Romero, R.: A first numerical simulation of the development and structure of the sea breeze on the Island of Mallorca, Ann. Geophys., 13, 981-994, 1995, http://www.ann-geophys.net/13/981/1995/.

Simpson, J.: Diurnal Changes in Sea-Breeze Direction, J. Appl. Meteorol., 35, 1166-1169, 1996.

Steyn, D. and Faulkner, D.: The climatology of sea-breezes in the Lower Fraser Valley, B.C., Climatological Bulletin, 20, 21-39, 1986.

Steyn, D. and Kallos, G.: A study of the dynamics of hodograph rotation in the sea breezes of Attica, Greece, Bound.-Lay. Meteorol., 58, 215-228, 1992.

Steyn, D., Ainslie, B., Reuten, C., and Jackson, P.: A Retrospective Analysis of Ozone Formation in the Lower Fraser Valley, British Columbia, Canada. Part I: Dynamical Model Evaluation, Atmos. Ocean, 51, 153-169, 2013.

Walsh, J.: Sea Breeze Theory and Applications, J. Atmos. Sci., 31, 2012-2026, 1974.

Yamazaki, H. and Satomura, T.: Nonhydrostatic Atmospheric Modeling Using a Combined Cartesian Grid, Mon. Weather Rev., 138, 3932-3945, 2010.

Zhang, Y., Chen, Y., Schroeder, T., and Kodama, K.: Numerical Simulations of Sea-Breeze circulations over Northwest Hawaii, Weather Forecast., 20, 827-846, 2005. 